\title{
"ENSEÑAR O CUIDAR A NIÑOS MALTRATADOS SOCIALMENTE": EL PROCESAMIENTO DE ESTE DILEMA EN EL MAESTRO DE UN ESPACIO EDUCATIVO ALTERNATIVO
}

\author{
Cecilia L. Durantini Villarino (Universidad de Buenos Aires)* \\ ceciliadurantini@hotmail.com
}

Recibido: 29/07/2011 Aceptado: 6/11/2011

\section{Resumen}

Los estudios institucionales preocupados por las dinámicas institucionales en condiciones críticas advirtieron que la situación de desempeño con niños maltratados socialmente puede configurarse para los docentes bajo el dilema "enseñar o cuidar". Aquí se presenta el modo en que el maestro de un grado creado para niños que no asisten a la escuela, procesó este dilema.

Esta cuestión se inscribe en una investigación que se pregunta por las condiciones y la dinámica de los Espacios Educativos Alternativos que favorecen, en los niños con experiencias de "fracaso escolar", el desarrollo de las posibilidades y los intereses por aprender.

Los resultados del estudio de caso mostraron que una de las condiciones que favorecían el aprendizaje de los niños era el estilo del maestro. El análisis de sus rasgos indica que el procesamiento que efectuó de tal dilema consistió en enfrentar la difícil situación de enseñar a estos niños sosteniendo su proyecto educativo. Este proyecto se orientaba a dar una respuesta educativa de carácter global y el modo de sostenerlo pareció consistir en la coexistencia compleja de la enseñanza y el cuidado al desarrollar la acción didáctica, organizativa, relacional y atencional.

\section{Palabras clave}

$$
\text { Estilo - Dilema - Enseñanza - Cuidado - Espacio Educativo Alternativo. }
$$

\section{Abstract}

The institutional studies interested on institutional dynamics in critical conditions found that the teacher's devolopment situation with socially maltreatmented children

* Licenciada en Ciencias de la Educación. Universidad de Buenos Aires. 
can appear for the teachers under the dilemma "teach or care". Here it is presented the way in wich the teacher of a grade created for children who are not at school, tried this dilemma.

This term is inscripted in a research that asks about the conditions and dynamics of the Alternative Educative Spaces that contribute, in the children with experiencies of schoolar failure, the devolopment of their possibilities and interests for learning.

The results of the study case showed that one of the conditions that contributes to the children's learning was the teacher's style. The analysis of his features showed that the kind of process that he made of such dilemma consisted in face the difficult situation of teach to these children, holding his educative project. This project was orientated to give a global answer and the way to sostain it seemed to consist in the complex coexistence of teach and care when he was devoloping the didactic, organizative, relational and attentional action.

\section{Key words}

Style - Dilemma - Teach - Care - Alternative Educative Space.

\section{Introducción}

Las políticas neoliberales de fines de los 90 que dejaron como saldo la precarización laboral y la desocupación, el crecimiento de la pobreza y la desestructuración del aparato productivo y del estado junto a los importantes movimientos migratorios en busca de mejores condiciones de vida desplegados ante estas crisis, fueron configurando un contexto especialmente turbulento (Schelemenson, 1987) para la vida institucional. En las escuelas, la intensificación y diversificación de las demandas de una población pauperizada; los bajos salarios docentes, la insuficiencia de recursos humanos y materiales junto a una imagen social desvalorizada, entre otros factores, concurrieron a desencadenar crisis de diferente amplitud cuyo impacto desestructurante sobre el espacio público y sus efectos desintegradores sobre el tejido social (Butelman, 1998) no han logrado repararse aún. Desde la línea de estudios dirigida por Fernández en el marco del Programa "Instituciones educativas" (IICE, UBA), que se preocupa por las dinámicas institucionales en estas condiciones críticas, se advirtió que la situación de desempeño con niños maltratados socialmente puede aparecer configurada para los docentes bajo el dilema "enseñar o cuidar".

Aquí se presenta el modo en que el maestro de un grado creado para niños que no están asistiendo a la escuela, ha procesado este dilema. Este aporte se inscribe en una investigación que se pregunta por las condiciones y la dinámica de los "Espacios Educativos Alternativos" (en adelante, EEA) que favorecen, en los niños que han vivido experiencias de "fracaso escolar", el desarrollo de las posibilidades y el despliegue de los intereses por aprender.

Un EEA es aquella respuesta del sistema educativo donde se hace posible que estos niños aprendan diferentes tipos de contenidos; adquieran una poten- 
cia efectiva para llevar adelante distintos aprendizajes (cognitivos, emocionales, sociales); se interesen por aprender y obtengan satisfacción al hacerlo. Se trata de un espacio que permite que los niños vivan las experiencias de aprendizaje que el "fracaso escolar" tiende a perturbar, como observan consistentemente los abundantes estudios al respecto (Luzuriaga, 1972; Molina García y García Pascual, 1984; Silva, 1993; Babini, 1994; Cordié, 1994; Thurlow 1995; Byrd, Weitzman y Avinger, 1997; Dawson, 1998; Nosei, y Lozano, 2001).

Los resultados del estudio de caso realizado mostraron que una de las condiciones que favorecían el aprendizaje de los niños era el estilo del maestro. Algunos de sus rasgos revelaron particularmente que el maestro no se resignaba ante la difícil situación de educar a niños maltratados y excluidos escolar y socialmente sino que la enfrentaba y sostenía su proyecto educativo de darles una respuesta educativa global. El modo de sostener este proyecto educativo pareció consistir en la coexistencia compleja (Morin, 2002) de la enseñanza y el cuidado al concebir y desarrollar la acción en cambio de oponer la enseñanza al cuidado.

En primer lugar, se sintetizan algunos de los hallazgos de los estudios institucionales citados. En segundo, se da una noticia sobre el enfoque epistemológico y el marco teórico de la investigación sobre EEA. En tercero, se presenta el caso. En cuarto lugar, se resume el diseño metodológico que informa su estudio. En quinto se describen los rasgos del estilo del maestro del caso de estudio. En último lugar, se plantea el modo en que este maestro parece haber procesado, según lo muestra su estilo, el dilema entre enseñar o cuidar a niños maltratados y excluidos escolar y socialmente.

\section{Los estudios de referencia acerca de la situación de desempeño con niños bajo maltrato y exclusión social}

Los estudios que aquí se toman como referencia en la comprensión de la dramática de las situaciones de desempeño con niños maltratados y excluidos socialmente corresponden a la línea de investigación sobre dinámicas institucionales en condiciones críticas llevada adelante por Fernández desde 1984, comenzando con el impacto de la masividad en las cátedras universitarias, luego con el niño pequeño en las escuelas infantiles y pasando por la educación en zonas aisladas. En particular la investigación efectuada en las escuelas primarias de un distrito escolar de la Ciudad de Buenos Aires (1995-2003) impactado por la llegada masiva de población migrante y el empobrecimiento a consecuencia de la pérdida de las fuentes de trabajo.

De acuerdo a sus hallazgos, el núcleo dramático que se activa en estas difíciles situaciones alude al temor a "estar frente a un niño dañado" a consecuencia del maltrato y el abandono social, y "a una posibilidad de reparación" a través de la tarea educativa puesta "en duda, cuando no imposible". Su activación intensifica las ansiedades típicas del vínculo pedagógico conduciendo a que la 
situación se configure en términos dilemáticos alrededor de la cuestión de "¿a qué dedicar el tiempo?". Bien a cuidar a los niños: dándoles cariño y alimento y reduciendo la violencia para formar un grupo, para luego poder enseñar pero perdiendo el tiempo de enseñar, para hacerlo más adelante. Bien a enseñar, que no es posible porque los niños están faltos de afecto, alimento, disciplina con lo cual también se pierde el tiempo de enseñar.

Ante este dilema, se identificaron diferentes tipos de procesamiento. En uno, el maestro tenía una visión cerrada e inmodificable de la situación y renunciaba a su posibilidad de acción. En otro, el maestro no se resignaba y enfrentaba la situación. En algunos casos, esto provocaba un sufrimiento que los anulaba transitoriamente o los llevaba a dejar la escuela o la zona. En otros casos, buscaban modos de sostener su proyecto educativo, con frecuencia recurriendo a concepciones pedagógicas, políticas o religiosas de sostén. En otro tipo de procesamiento, generalmente los de maestros en situación personal de vulnerabilidad -embarazo, juventud, proximidad al retiro-, trataban de cubrir los vacíos sufridos por sus alumnos.

\section{Noticia sobre el Enfoque Epistemológico y el Marco Teórico}

A nivel epistemológico se parte de la hipótesis de la complejidad de los fenómenos de la realidad (Morin, 2007), es decir de su carácter incierto, contradictorio y a la vez complementario, recursivo y hologramático. De allí la necesidad de un pensamiento fundado en los principios de distinción, conjunción e implicación tal como Morin los entiende, que tome como objeto una intersección de relaciones y problemáticas diferentes y en transformación. Ésta hipótesis justifica la decisión de adoptar un enfoque multirreferencial que propone, básicamente, implementar lecturas plurales, sistemas de referencia distintos para comprender el objeto en los siguientes niveles de inteligibilidad: el de la institución, la organización, el grupo, las interrelaciones y las personas (Ardoino, 2005).

En lo que respecta al marco teórico, aquí se detallan las perspectivas de mayor relevancia para el tema sobre el que se hace foco.

Desde el psicoanálisis, Winnicott plantea que el desarrollo de un ser humano integrado tiene lugar cuando se han provisto las condiciones adecuadas para ello y éstas se derivan de las que reúne una madre suficientemente buena y un buen hogar.

La "madre suficientemente buena" cuida del niño con una técnica personal basada en la comprensión de lo que necesita, a través de la identificación con él y de la interpretación de sus señales. Esta madre no somete al niño a la propia impulsividad, temores, ansiedades y rabias. Por eso, su cuidado equivale a una prueba de confiabilidad personal cuya continuidad en el tiempo da al bebe un sentimiento de confianza en esta madre, primero, y en el ambiente, después. Para proporcionarlo, la madre debe lograr que el hijo sienta que ella es una 
persona viva con objetos internos vivos que no se oponen al estar vivo del niño. Bajo estas condiciones, él puede interiorizar los elementos del cuidado materno convirtiéndolos en apoyos de su ser en formación.

El primer elemento es, para Winnicott, el sostenimiento. Éste abarca, por una parte, todo manejo físico que evite la afrenta el niño con el medio externo gracias a la adaptación materna a las necesidades del bebe. Por otra parte, aspectos más complejos como, por ejemplo, la visión del niño como una persona antes de que lo sea. Con lo cual la madre se constituye en un medio que contiene y posibilita la integración de la experiencia infantil.

El segundo es la presentación del mundo externo mediante una adaptación cuantitativa y cualitativamente activa al niño que satisfaga las necesidades de dependencia y ofrezca oportunidades para la independencia.

También es importante la supervivencia materna a la agresión infantil que se manifiesta en el mantenimiento del mismo amor y los mismos principios, sin tomar represalias. Así como en la indulgencia ante los reclamos del niño por las frustraciones resultantes de las deficiencias en su cuidado.

Finalmente, Winnicott señala que, cuando la madre suficientemente buena mira al hijo, su rostro se relaciona con lo que ve en él, permitiéndole verse a sí mismo. En ese sentido, mirar a la madre y ser visto por ella constituye una experiencia de autodescubrimiento y de descubrimiento de significados en la realidad externa.

Otro aporte del psicoanálisis pertenece a las elaboraciones de Ulloa (1995, $2001,2005)$ sobre la crueldad y la ternura. Desde su perspectiva, la crueldad es un dispositivo socio cultural cuya expresión típica es la encerrona trágica. Esta situación de dos lugares (víctima y victimario) se configura cada vez que alguien, para cubrir sus necesidades, depende de alguien que lo maltrata, sin posibilidad de apelar a un tercero. La crueldad se origina en el fracaso del primer amparo al que todos están destinados: la ternura.

La ternura es el escenario donde ocurre el pasaje de cachorro humano a sujeto social, pulsional y ético y su paradigma se halla en la ternura materna. Una de sus producciones ejes es la empatía que garantiza el suministro de lo necesario para el hijo. Otra, el miramiento que evita los abusos y permite el desprendimiento hacia la autonomía al mirar con considerado interés a quien, habiendo salido de sí, se reconoce como un sujeto distinto. Por eso. En ese sentido, bajo la impronta de la ternura, señala Ulloa, el sujeto no solo es hechura de la cultura sino también su hacedor. Los tres suministros básicos de la ternura a lo largo de la vida son "el abrigo para los rigores de la intemperie; el alimento para los del hambre y el buen trato ante las inclemencias del vivir" (Ulloa, 1995).

Desde el sociopsicoanálisis, uno de los principales aportes de Mendel se halla en el concepto de acto poder como aquel que modifica -constructiva o destructivamente- el medio social. Al producir efectos sobre el medio, el acto 
opera como un medio de autonomización parcial del sujeto respecto de las presiones de la realidad interna y externa que le confirma su poder sobre si y sobre el mundo donde vive. Esto ocurre cuando el sujeto ejerce poder sobre sus propios actos. Si no puede hacerlo, el individuo pierde posibilidades de verificar sus efectos sobre la realidad externa. Entonces se desinteresa por lo que hace y experimenta un mayor sentimiento de imposición externa, fuente de displacer. En términos de Mendel, "se descubre impotente para realizar un acto preciso y personal" (1974, b, p. 107) porque "el poder sobre la realidad y el entronque con lo real que caracterizan el acto se han perdido" (Mendel, 1974 , b, p. 106). El cansancio es la única prueba de sus actuaciones. Por eso, el sujeto siempre desea ejercer poder sobre sus actos y cada vez que lo logra, actualiza la potencialidad de construir personalmente su historia.

Otro de los aportes de Mendel reside en su perspectiva acerca de la firmeza en oposición al fenómeno de la autoridad. La firmeza consiste en "permanecer siendo uno mismo portador explícito del propio sistema de valores que se sostiene frente al otro considerado como interlocutor válido" (Mendel, 2004 , p. 51). Esta actitud permite al docente asumir una dimensión parental en el desempeño de su rol, dando respuesta a las demandas afectivas de los alumnos, sin convertirse en sus padres.

Desde la teoría de la acción, los trabajos de Barbier estudian la acción humana entendida como “... la movilización de una combinación inédita de rutinas. La noción de rutina (interiorización de una secuencia de actividad) y la noción de inédito (combinación no experimentada) carecen de sentido fuera de la relación con el actor implicado, y particularmente con sus experiencias anteriores y en relación con las supuestas huellas (estructuras operativas) que ellas han dejado en su personalidad..." (Barbier y Galatanu, 2000, p. 4). El esquema de análisis de la acción que propone hace foco en el objeto a partir del que se efectúa la acción, los medios movilizados, el actor o los actores implicados, las relaciones establecidas entre ellos y con el objeto, los resultados y sus efectos, deteniéndose especialmente en la consideración de los lazos entre acción e identidad.

Para Barbier (1997), la relación entre la acción y las imágenes de sí se establece mediante un proceso de interiorización de las experiencias vividas por el sujeto, en sus componentes afectivos, representacionales y operatorios. Este proceso se acompaña de la vivencia de afectos y la producción de representaciones acerca del entorno de la acción, la acción y el sujeto actuante.

Desde el análisis institucional, resultan de especial interés las conceptualizaciones de Fernández (1997, 2002, 2006, 2007) sobre dramática institucional y núcleo dramático.

La primera refiere al suceder tal como lo viven, lo sienten los sujetos en diferentes planos, el real visible; el emocional no visible, conciente e inconciente, y el emocional más profundo. La segunda a "la particular formulación de un 
conflicto que subyace en los niveles no manifiestos como principal atribuidor de sentido" (1998, p. 141). Este conflicto se vincula con la significación que adquiere la educación como mandato social y como respuesta a las necesidades del sujeto y suele quedar expresado en los términos de un planteo dilemático. Por lo cual posee potencial para generar ansiedad y se expresa en aspectos centrales del movimiento institucional manifiesto, particularmente en los sobre esfuerzos organizacionales dirigidos al control de los comportamientos y las condiciones institucionales básicas. En los espacios educativos, el núcleo dramático parece concernir a la vida y muerte social de los sujetos en formación. En condiciones críticas, a la lucha de un grupo por mantener con vida o dejar morir a otros que la sociedad ha puesto a su cargo y, por lo demás, ha abandonado (2006, p. 37) en principio a la muerte social.

La noción de crisis (Kaës, 1979; Thom, 1976) alude a acontecimientos sociales excepcionales, vividos como un quiebre de la experiencia y como una interrupción de la continuidad del sí mismo, que disminuyen la capacidad de los mecanismos de regulación habituales para responder a las exigencias del ambiente. En las instituciones educativas, las condiciones críticas suelen estar dadas por los cambios sociales que afectan a las poblaciones atendidas y por la conmoción de los modelos y estilos institucionales. Su combinatoria conduce a la desorganización de las relaciones y a estados subjetivos de ansiedad y pérdida de sentido de la tarea y la pertenencia que se ven intensificados por la activación del núcleo dramático, en reposo durante otros períodos de la vida institucional. En esta situación, la ansiedad se deposita con frecuencia en condiciones institucionales básicas como el tiempo o el espacio. Con lo cual aumenta su sobre manipulación mediante la creación de normas y la producción de ideologías que racionalizan tales comportamientos.

El último aporte de relevancia aquí es el de Cols (2011) sobre el estilo de enseñanza definido como una "particular combinación de rasgos en el modo de concebir y llevar a cabo la enseñanza" que se expresa en las tres dimensiones de la acción docente. La dimensión didáctica refiere a la estructuración del conocimiento y la promoción del aprendizaje del alumno y de su acceso a formas culturales contenidas en el curriculum. La organizativa se centra en la definición y el sostén del encuadre de trabajo y el manejo de la clase. La dimensión relacional o pedagógica, en el logro y el mantenimiento de la relación pedagógica y en el acompañamiento y el sostén emocional del alumno en el proceso de aprendizaje. El estilo no resulta de la sumatoria de rasgos independientes referidos a cada dimensión sino de una singular combinación que aparece como recurrente a través del análisis de la actuación en clase y el discurso del actor acerca de su acción (Cols). 


\section{EI Caso de Estudio}

El caso de estudio elegido fue uno de los grados perteneciente al Proyecto Grados de Nivelación que funciona desde el año 2000 como parte de la oferta del sistema educativo común y público de la Ciudad de Buenos Aires. Este proyecto se dirige a los niños que nunca fueron o abandonaron la escuela primaria y propone reintegrarlos a la escuela común en el grado más próximo a su nivel de aprendizaje y edad o bien a una instancia más adecuada a su situación escolar. Cada grado de nivelación pertenece a una escuela primaria común, funciona en jornada simple de lunes a viernes y puede ubicarse dentro o fuera del establecimiento escolar. El plan de trabajo contempla la articulación del trabajo en el grado con la participación de los niños en actividades (música, educación física, actos, excursiones, etc) que se acuerden con la escuela de referencia.

En la zona donde se sitúa el grado de nivelación (en adelante GN) caso de estudio, una sola escuela contaba con estos grados. Esta escuela atendía aproximadamente a 900 alumnos distribuidos en 30 secciones, 250 de ellos en condición de sobreedad.

En el año 2006, el GN caso funcionaba como primer ciclo en el turno tarde, en el aula de un Centro Comunitario ubicado en una villa de emergencia próxima a dicha escuela. El grupo de niños contaba con 9 varones y 3 nenas, de 6 a 13 años. Eran argentinos 5 niños, paraguayos 3 y bolivianos 4 . Sus condiciones de vida correspondían a las de un hogar con necesidades básicas insatisfechas según los indicadores de privación del INDEC. Las trayectorias socio familiares aparecieron fracturadas por acontecimientos que interrumpieron la continuidad de la vida familiar, desestabilizaron la vida del grupo y dificultaron la escolaridad debido a que afectaron la asistencia, el rendimiento y el comportamiento del niño. Entre ellos, las migraciones, las adicciones y el fallecimiento de hermanos, la separación de la familia y el abandono.

La mayoría de ellos (9) habían asistido a una escuela pero los rechazaron por no tener dni, los derivaron por su mala conducta o desertaron luego de repeticiones reiteradas. Mientras que 3 niños no fueron nunca a la escuela.

El maestro del GN caso se desempeñaba en el cargo desde el año 2004. En el año 2003, el supervisor del distrito presenció un acto diseñado por él para el día del maestro donde se reunían los personajes de los cuentos tradicionales "muchos años después". Al año siguiente, este supervisor, a quien le interesó mucho su propuesta, que era varón y pertenecía al Programa Zap, lo llamó a su casa para invitarlo a tomar un GN. El maestro estaba indeciso, algunas personas le recomendaban no tomar el cargo porque se disolvería con el proyecto, los niños tenían problemas de conducta y los GN funcionaban en la villa. Otras lo animaban a hacerlo considerando que el supervisor mismo lo invitó. Finalmente, él decidió renunciar a su cargo como preceptor titular e incorporarse al GN. 
Su trayectoria escolar comenzó en un jardín de infantes y continuó en una escuela pública donde sus padres participaron de la cooperadora. También asistía a clases particulares de dibujo. El maestro recordó la exigencia de su mamá para que fuese "prolijo" y el rechazo y la obediencia con que él vivía sus castigos. Cuando estaba en $7^{\circ}$ grado, encontró en la vereda de la escuela muchos libros de la biblioteca para tirar a la basura. Entonces decidió llevarlos a su casa. Más tarde le regaló uno a una profesora del secundario.

El secundario lo realizó en una escuela pública. Al finalizarlo, dudó entre la carrera de letras y formaciones más breves para continuar después con otros estudios. En primer lugar, realizó el Profesorado de Nivel Primario en el Normal 3, el de Nivel Inicial en el Normal 11 y el de Adultos en el Colegio Marista. En esta época también realizó una formación en el Instituto de Bellas Artes.

Su trayectoria profesional comenzó como maestro de nivel primario en una escuela privada a la que renunció debido a que no le efectuaban los aportes sociales descontados del sueldo. Entonces, el dueño del colegio lo amenazó con cerrarle otras fuentes de trabajo si lo denunciaba. Así dio inicio a su trayecto en el circuito público de Ciudad de Buenos Aires, temeroso de no conseguir cargo por su bajo puntaje. Hasta el año 2005 se desempeñó como maestro de primer ciclo en el proyecto "maestro + maestro" del Programa Zap. Allí sentía que su tarea se aproximaba a la de un celador debido a que había otro docente a cargo del grado y que la escuela empobrecía su funcionamiento.

El maestro también trabajó en otros niveles del sistema educativo. Se desempeñó como preceptor de nivel medio y como docente de geografía en un secundario de adultos, donde encontró que los estudiantes mostraban buena disposición y ellos apreciaron su dedicación. En las colonias de nivel inicial de verano e invierno de la Ciudad de Buenos Aires, fue preceptor, coordinador de grupo y de colonia. De esta experiencia, apreció el contacto con personas del ámbito recreativo y artístico que le aportaron propuestas novedosas.

En el 2006, año del estudio de caso, el maestro se desempeñaba por tercer año consecutivo en el GN del turno tarde. Su experiencia hasta aquí había sido lo suficientemente gratificante como para continuar allí. Ese año también tomó cargo como maestro titular de primer grado en el turno mañana de una escuela en provincia de Buenos Aires. La escuela estaba ubicada en una villa de emergencia. Los vecinos, en su mayoría inmigrantes bolivianos, venían demandando su apertura desde años atrás. Su grado se componía de 40 alumnos que no fueron al jardín de infantes. El maestro relató que obtuvo un pizarrón, lo pintó y colocó con la ayuda de su padre. Se sentía entusiasmado con la escuela porque era nueva y había que "empezar todo de 0 , a pulmón".

\section{Noticia sobre el Diseño metodológico}

A nivel metodológico, el estudio descriptivo (Selltiz, 1965) de caso (Saltalamacchia, 2000) combina los encuadres (Bleger, 2003) situacional e histórico 
siguiendo la propuesta metodológica de Fernández L. M. (2006) para el abordaje de lo institucional, e incorpora aportaciones de la etnografía (Rockwell, 2009). Su enfoque clínico institucional supuso un diseño basado en ciclos de contrato -con los sujetos del caso-; indagación diagnóstica; descripción analítica de los materiales; producción de informes de avance, validación de los avances en reuniones de devolución y trabajo con los sujetos implicados; nuevos análisis; consultas y revisiones teóricas; reformulación del problema y reiteración del ciclo hasta la producción del avance final. Su desarrollo involucró tres fases de trabajo en terreno.

Durante la "Fase de Exploración" (año 2005, 16 horas en terreno) se consultó a informantes claves y se observó la vida cotidiana del caso de estudio, también se revisó un estudio exploratorio previo. En la "Fase de Indagación" (2006, 300 horas en terreno) se trabajó con los actores del GN caso y de las organizaciones vinculadas al mismo, la vida cotidiana y los documentos normativos. Las fuentes secundarias abarcaron los informes de las investigaciones antecedente directo de este estudio. Durante la "Fase de Devolución y Validación" (2007, 30 horas en terreno) se consultó al maestro y a los niños del GN caso.

Los instrumentos empleados fueron las observaciones de carácter no estructurado (jornadas completas, de temas específicos y abiertas de impregnación); las entrevistas institucionales (de indagación, profundización y elaboración); los dispositivos proyectivos expresivos (el dibujo de escenas, la narración de una fábula y la construcción de un mapa); las pruebas de rendimiento en lectura y escritura y en matemática y el registro y la recolección de documentos primarios (disposiciones normativas, cuadernos de los niños, planificaciones del maestro entre otros) y documentos secundarios consistentes en informes de avance de investigaciones antecedentes en el distrito.

El diseño de análisis articuló fases de aproximaciones sucesivas a cada tipo de material obtenido en terreno con su triangulación. Cada aproximación comenzó con la lectura abierta y en profundidad del material y fue seguida por un trabajo descriptivo que permitió armar documentos de base compuestos por los sistemas de categorías, los materiales categorizados; la medición de las dotaciones de las categorías; las descripciones analíticas (Rockwell, E, 2009) y de síntesis y periodizaciones y representaciones gráficas. La tarea interpretativa hizo foco sobre los significados que los sujetos daban a los fenómenos desde su experiencia vivida. La comprensiva, sobre la formulación de relaciones entre los fenómenos estudiados y con las configuraciones interpretativas elaboradas (Blanchard Laville, 2005). A través de este proceso se construyeron propuestas conjeturales integradas por proposiciones con diferentes grados de amplitud. Éstas fueron sometidas constantemente a la revisión de su consistencia lógica y a la constrastación con el corpus teórico y empírico (Mancuso, 2008). Lo cual permitió precisar sus fundamentos y validarlas hasta la elaboración de las conclusiones finales sobre los resultados del caso. 
Para el estudio del estilo del maestro del GN caso se aplicó este diseño a las crónicas de observación de las clases y a los testimonios de las entrevistas y de las conversaciones espontáneas con él. Las entrevistan comprendían las de indagación tomadas al inicio y la mitad del año; la entrevista de elaboración efectuada al cerrar el año y las entrevistas de profundización del material de crónicas correspondientes a los tres momentos del año recién distinguidos.

A través del trabajo con estos materiales se fueron construyendo líneas de trayectoria biográfica, escolar, académica y profesional; gráficos de ubicación y desplazamiento en el espacio; descripciones densas y cuadros de análisis cuantitativos, entre otros materiales.

Los avances en el análisis se presentaron sucesivamente al maestro en las entrevistas de profundización y en la entrevista de devolución y validación efectuada en el año 2007. Sus reflexiones, preguntas y comentarios sirvieron al ajuste de la propuesta interpretativa final.

Ésta se organiza en torno a la trayectoria, las concepciones y los rasgos de la acción del maestro. A nivel teórico está informada por las conceptualizaciones de la acción de Barbier y la propuesta de Cols (2011) sobre el estilo docente. A las dimensiones didáctica, organizativa y relacional que la autora propone se agregó aquí la dimensión atencional, referida al adecuado mantenimiento del entorno material, la provisión de recursos y la asistencia física del niño.

\section{El estilo del maestro del GN caso de estudio}

Uno de los rasgos de su estilo era el sostén (Winnicott) del niño dado, en principio, por la hipótesis de partida sobre sus posibilidades de aprender y de interesarse por su aprendizaje, sin contar con referencias ni evidencias al respecto. Luego, contando con este marco, por el establecimiento de una relación donde, por una parte, se hallaba reducido lo imprevisto (por el cumplimiento de los acuerdos establecidos con el grupo: por ejemplo, anticipar una ausencia y explicarles sus causas; traer un tipo de material -libros, fideos de letras- o realizar una actividad determinada -lectura o dibujo). Por otra, se protegía al niño de los imprevistos del medio externo (mediante la anticipación y planificación de sucesos fuera de lo habitual como una excursión o un acto y la explicación de los eventos no planificados) y de los contactos que dañasen la imagen de sí de los niños o interrumpieran los procesos en curso. Por ejemplo, a principio de año, el maestro suspendió las clases de plástica con un grado común en la escuela debido a que el profesor puso en penitencia en un rincón a uno de los niños del GN -que no dejaba de llorar- por no prestar atención. En cambio, mantuvo las clases de informática y biblioteca donde las maestras preparaban proyectos y recibían a los niños del GN con entusiasmo por integrarlos.

Otro rasgo era la ternura (Ulloa) fundada en el reconocimiento de cada niño como sujeto singular (su forma de ser, sus tiempos, sus preferencias, su forma de actuar), social (perteneciente a una familia, una cultura, un sector so- 
cial determinados) y de aprendizaje (con posibilidades y derecho de aprender) y en el amor hacia ellos. Sobre estas bases, las acciones del maestro (animar la colaboración en las consignas de trabajo, colocar música, bromear si había tensión entre los niños, por ejemplo) constituían respuestas pertinentes y solícitas a las necesidades de abrigo, alimento y buen trato material y simbólico de los niños que tomaban en cuenta sus avances y logros.

De una manera tal que, como señala Winnicott, les devolvía una imagen de sí mismos donde ellos podían descubrirse. Por ejemplo, en una ocasión, Lucas, uno de los niños, observaba que el maestro le mostraba a su compañero más pequeño una imagen y le preguntaba pacientemente por segunda vez “¿qué es esto Ramón?” sin obtener respuesta. Lucas impaciente exclamó "¡Dale! ¡Hablá!". El maestro, con la misma paciencia le dijo "es chiquito, no habla castellano, está aprendiendo". Aquí el maestro transmitía a Lucas que aprender "lleva tiempo", el tiempo que le dedicaba a Ramón como el que le dedicaba a él para explicarle el proceso de Ramón.

Este ejemplo muestra también la firmeza (Mendel) del maestro. Entendida en primer lugar, como la coherencia entre su esquema de valores, su proyecto educativo, el modo de llevar a cabo la acción, y, los comportamientos y los valores que alentaba en los niños. En segundo, como la presentación de sus fundamentos a ellos tomándolos como interlocutores válidos. En tercero, como el mantenimiento de su posición frente a las agresiones de los niños y a los inconvenientes con las organizaciones del medio externo.

Su estilo también se distinguía por la seriedad como maestro a cargo del GN. Por el compromiso con el proyecto de educar a estos niños y la dedicación puesta en el desarrollo de la acción en sus diferentes dimensiones. Por ejemplo, el primer día de clase, mientras barría y ordenaba el aula del Centro Comunitario -sacaba un espejo roto, juntaba recipientes vacíos, colocaba una bolsa en uno para usar como basurero y quitaba las cortinas descoloridas- el maestro describía las acciones realizadas, mencionaba sus fines (evitar que se lastimen o ensucien sus guardapolvos nuevos y limpios), anticipaba otras (traería cortinas nuevas, un recipiente para lápices de colores y tijeras) y solicitaba colaboración para completar la preparación del aula (traer diarios y revistas para recortar palabras, jabón para lavarse las manos, rejilla para las mesas). Los niños lo observaban con atención y respondían tímidamente a sus pedidos levantando la mano.

Su estilo se caracterizaba además por la adaptación (Winnicott) a las necesidades, las formas y el proceso de cada niño y del grupo, especialmente en lo relativo al aprendizaje. El maestro mantenía la atención flotante sobre el suceder, dispuesto a percibir las demandas manifiestas y no manifiestas y a identificar los cambios. Con lo cual iba adecuando, cuantitativa y cualitativamente, el desarrollo de la acción tanto a los obstáculos y las frustraciones que iban enfrentando los niños como a los avances y logros que mostraban. 
El estilo del maestro se distinguía, finalmente, por la elaboración de las dificultades, los sucesos y los temas que provocaban frustración, temor, ansiedad o sufrimiento, tratándolos mediante la palabra y devolviéndolos con significado a los niños.

Estas selecciones de testimonios de entrevistas y de crónicas de observación muestran la expresión combinada de los rasgos recién identificados en el estilo de este maestro.

... creo que en algún lugar las cosas llegan, de eso si estoy seguro y que también tenés que poner una ficha en el chico y apostar en algo... en ellos, aunque sea algunos cambios si se pueden hacer, se pueden lograr, estoy convencido (testimonio de entrevista de indagación).

... lo que pienso siempre... que cada día que ellos vengan... aprendan algo, que se lleven algo y que les haya servido de algo haber estado, que no haya pasado porque sí... Por ejemplo, el día que fuimos a ver a ver "Ópera Pampa" les puse "Ayer fuimos", como que quede constancia de eso también, por escrito y que ellos también que ese día no fue porque sí, que estuvo... a mí me parece que lo bueno o lo importante del tiempo que estuvieron es haberle dado lo mejor, que hayan podido aprender algo... que el tiempo que estuvieron no estuvo desperdiciado (testimonio de entrevista de profundización).

... yo lo conozco a cada uno, sé los que les pasa, cómo están, más allá de que a veces lo haga ver o no... yo soy muy observador... me absorbe mucha energía pero quizás tiene que ver conmigo que veo todo... y ellos también lo ven... (testimonio de entrevista de profundización).

...tenían que hacer una tarea para el otro día, todos me la traen menos Erwin, entonces le preguntaba ¿por qué? Y se quedaba mudo. 'No te estoy retando, quiero saber porqué no la hiciste, así entiendo porqué no la pudiste hacer... una cosa es que te duermas, otra cosa es que te fuiste a jugar a la pelota...y otra cosa es que te fuiste con tu novia'... y ahí se mataron todos de la risa... En realidad apunto a eso, a que hablen, a que puedan fundamentar...a veces les digo '¿por qué?' y se me quedan así mirando... Son inteligentes porque son todos inteligentes, los chicos son mucho más vivos que uno y ellos esperan que el otro los va a retar...yo no tengo problema...está bien, no pudiste pero explicame porqué no lo hiciste. Igual le puse una nota, le corregí a todos y abajo le puse 'acá te pongo esto -le dije- no hizo la tarea, pero que le puse: porque se quedó dormido, la próxima vez para que lo sepas'. Se fue muy contento, se llevó la nota y chau... pensativo ¿viste?.... (testimonio de entrevista de profundización).

Erwin serio, con las piezas del rompecabezas que intenta armar por segunda vez -ya que el primero no le salió- desparramadas sobre el cuaderno 
y algunas en el piso, no levanta la mirada de su cuaderno -parece vencido-. El maestro observa y luego apoya la mano sobre el botón del ventilador, mira a los niños (voz alta).

-¿Puedo apagar el ventilador un poco?... Porque se le vuela todo a él ¿puedo?...

Se oyen "si" en voz bajita. (crónica de observación, abril).

El maestro toma el cuaderno de Jonny y lo muestra, golpea la tapa con sus nudillos

-Mira...un cuaderno lindo...Igual como todos los cuadernos, pero digo... El papá o la mamá de ustedes que compró esto lo hizo con sacrificio, así que cuídenlo porque después empiezan a perder las cosas... ¿Si Jhony? (cariñosamente) ¡Muy bien que empezes así, eh!

Jonny mira hacia abajo sonriendo, parece contento con las palabras del maestro y también avergonzado.

El maestro continúa.

-Todos muy bien... Carlitos también... todos empezaron muy bien... La caja que me olvidé de pedir, voy a ver si mañana paso... una caja con materiales que vamos a tener... (tono serio) Pongánse las pilas, practiquen, estudien... así que vengan... traten de venir... (baja el tono de voz) obviamente que a cualquiera le puede pasar, me siento mal, medio enfermo, algo... pero sino, traten de venir, estudiar... (suave y firme) Hay que empezar bien ahora... ponerse las pilas, apurarse... entonces ya agarrarle la mano bien de entrada...esto depende mucho de ustedes, ¿eh muchachos?... yo de acá, hago mi parte... "copien"... y yo miro para otro lado, y llego a casa y no hago nada... no aprendo... (crónica de observación, marzo).

Los niños están parados frente a la puerta, el maestro está atrás de ellos, los mira, cariñosamente, sonriendo

-Bueno, hemos pasado este primer día... ¿contentos?

Los niños asienten con la cabeza, miran hacia delante, parecen incómodos o avergonzados, con timidez.

El maestro (sonríe).

-Yo estoy contento... (crónica de observación, marzo)

\section{Enseñar o cuidar o "Enseñar es cuidar y cuidar es enseñar"}

El estudio del estilo de este maestro permite plantear que, ante el impacto producido por el maltrato y la exclusión social de los niños del GN, él ha efectuado el tipo de procesamiento que se caracteriza por no resignarse y enfrentar la situación buscando modos de sostener su proyecto educativo.

Este proyecto parecía orientado a dar a los niños un buen presente, en sus términos, "que estén bien, que la pasen bien" en el GN: escuchando música, leyendo libros, fabricando máscaras, con tiempo para aprender y para jugar. También se dirigía a proporcionarles herramientas "para que vuelvan a la 
escuela" y "para que se defiendan en la vida. Por ejemplo: que lean, escriban, operen matemáticamente, adquieran habilidades productivas (fabricación y reparación de objetos) y sociales (comunicarse apropiadamente, obtener y dar información) y desarrollen la capacidad de atender, advertir riesgos, planificar y decidir evaluando los distintos factores en juego. En este sentido, a su modo de ver, estos niños en riesgo necesitaban una respuesta educativa de carácter global.

De acuerdo a los análisis efectuados, es posible pensar que, uno de los principales modos de sostener este proyecto educativo consistió en la coexistencia compleja (Morin) entre enseñar y cuidar, haciendo de la enseñanza, cuidado y del cuidado, enseñanza al concebir y desarrollar la acción en sus diferentes dimensiones -didáctica, organizativa, relacional y atencional-. En otras palabras, en el mantenimiento de las relaciones complementarias y a la vez antagonistas (Morin) entre enseñar y cuidar. El maestro parece haber logrado esa coexistencia haciendo de la enseñanza, cuidado y del cuidado, enseñanza.

Al enseñarles a los niños los contenidos escolares, las pautas de un trabajo bien hecho (correcto, prolijo, bello), las normas para relacionarse con otros adultos, las precauciones para evitar conflictos que interrumpan los procesos de aprendizaje en curso, observando, respetando y adecuándose a las necesidades, intereses, posibilidades y estilos de los niños; el maestro los estaba cuidando.

El cuidado, retomando los términos de Winnicott, se basa en la comprensión de las necesidades del niño, es personal y reúne entre sus principales elementos el sostenimiento y la presentación adaptativa del mundo externo. Por eso, puedo decirse que el maestro también cuidaba a los niños cuando cumplía con las tareas que les presentó como su responsabilidad (explicar las actividades, llegar a horario, corregir, realizar la excursión, dar clase de dibujo), fundamentaba sus decisiones (en cuanto a la organización del tiempo y el espacio, la continuidad de un tipo de actividad), los defendía de malos tratos en la escuela y les mostraba los pasos, herramientas y modos con que llevaba adelante sus acciones. Al cuidarlos de estas maneras, el maestro les estaba enseñando el valor de la responsabilidad, la necesidad ética de dar cuenta de los actos, su derecho a un trato digno y a la educación y la existencia de alternativas para actuar.

Desde esta perspectiva, su estilo hacía posible que cuando le enseñaba al niño lo estuviera cuidando y que cuando lo cuidaba, le estuviera enseñando. Gracias a ello, pareció lograr escapar a los efectos desviadores y paralizantes que el dilema entre enseñar y cuidar tiene sobre la acción, en estas situaciones de desempeño profesional. En términos de Mendel, podría decirse que este maestro ejercía poder sobre sus actos. 


\section{Referencias Bibliográficas}

- $\quad$ Ardoino, J. (2005). Pensar la educación desde una perspectiva epistemológica (1 Ed.). Buenos Aires: Novedades educativas.

- Barbier, J. M. y Galatanu, O. (1997). Algunos lazos entre acción, afectos y transformación de sí. S.d.

- Barbier, J. M. y Galatanu, O. (2000). La singularidad de las acciones: algunas herramientas de análisis. S.d.

- Butelman, I. (1998). Pensando las instituciones. Buenos Aires: Paidós.

- Fernández, L. (s.d) Dinámicas institucionales en situaciones críticas: el impacto sobre las prácticas educativas con niños sometidos a maltrato y extrema marginalización social. Avances de investigación. Revista IICE, nº 20 pp18-27 Buenos Aires. Miño Dávila.

- Fernández, L. (2006). Espacios institucionalizados de la educación. Algunos componentes nucleares en la identidad institucional y su consecuencia para el análisis En Monique Landesmann (Coord.) Instituciones Educativas: Instituyendo disciplinas e identidades (pp 29-60). México: Casa Juan Pablos.

- Manoni, M. 1967. (s.f). El niño, su enfermedad y los otros. Buenos Aires: Nueva visión.

- Manoni, M. (2000). La educación imposible ( Ed.). México: Siglo XXI.

- Manoni, M. Un lugar para vivir. S.d.

- Mancuso, H (2008). Metodología de la investigación en ciencias sociales. Buenos Aires: Paidós.

- Mendel, G. (1974, a). Sociopsicoanális 1. Buenos Aires: Amorrortu.

- Mendel, G. (1974, b). Sociopsicoanálisis 2. Buenos Aires: Amorrortu.

- Mendel, G. (2004). Sociopsicoanálisis y educación. Buenos Aires: Novedades Educativas.

- Morin, E. (2007). Introducción al pensamiento complejo. Buenos Aires: Gedisa.

- Rockwell, E. (2009). La experiencia etnográfica (1 Ed). Buenos Aires: Paidós.

- Selltiz, J. Deutsch y Cook (1965). Métodos de investigación en las ciencias sociales. Madrid: Realp.

- Ulloa, F (1995). Novela clínica psicoanalítica. Historial de una práctica. Paidós, Bs.As.

- Ulloa, F (2004). Sacrificio, ternura /crueldad y poder soberano.

- Ulloa, F (2005): Sociedad y crueldad. Seminario Internacional. La escuela media hoy. Desafíos, debates, perspectivas. Panel Brecha social, diversidad cultural y escuela. Ministerio de Educación, Dirección Nacional de Gestión Curricular y Formación Docente.

- Winnicott, D. W. (1990). Deprivación y delincuencia. Buenos Aires: Paidós.

- Winnicott, D. W. (1993). El niño y el mundo externo. Buenos Aires: Horme. 